\title{
Comunicar con los visitantes: una 'nueva' faceta profesional para los conservadores-restauradores
}

\author{
Santos M. Mateos Rusillo
}

Resumen: Los recursos patrimoniales están más expuestos que nunca a la interacción con los visitantes. Por tanto, ya no basta con trasladar a un público mayoritario unos determinados contenidos culturales, pues la actual situación demanda la inclusión de un tipo de mensajes que fomenten y favorezcan la relación recursovisitante de forma sostenible. Y es precisamente la Difusión preventiva, entendida como una estrategia de sensibilización para informar y persuadir a los visitantes de la extrema fragilidad de los recursos patrimoniales, la que lo permite de forma óptima.

Además, utilizar esta estrategia es una magnífica herramienta al servicio de la identidad corporativa de los propios recursos patrimoniales y de sus conservadores-restauradores. Normalmente, muchas cuestiones relacionadas con las políticas y acciones de conservación preventiva no se explicitan a los visitantes, pero al integrarse en el discurso que se les traslada, permite que estos tengan una idea cabal de esos esfuerzos, lo que redunda en la imagen que tienen del recurso en cuestión y, por extensión, de sus conservadoresrestauradores.

Palabras clave: Patrimonio cultural; Museo; Conservador-restaurador; Conservación preventiva; Difusión preventiva; Visitante.

\section{Comunicar com os visitantes: uma 'nova' faceta profissional para os conservadores-restauradores}

Resumo: Os recursos patrimoniais encontram-se mais sujeitos do que nunca à interacção dos visitantes. Deste modo, não basta divulgar determinados conteúdos culturais à maioria do público, pois a situação actual obriga à inclusão de um tipo de mensagens que fomentem e favoreçam a relação recurso-visitante, de forma sustentável. E é precisamente a Difusão preventiva, entendida como uma estratégia de sensibilização para informar e persuadir os visitantes da extrema fragilidade dos recursos patrimoniais, que a permite optimizar.

Além disso, a utilização desta estratégia é uma magnífica ferramenta ao serviço da identidade corporativa dos próprios recursos patrimoniais e dos seus conservadores-restauradores. Normalmente, muitas questões relacionadas com as políticas e acções de conservação preventiva não se explicam aos visitantes, mas integra-las no discurso que lhes é transmitido permite-lhes ter uma ideia cabal desses esforços, resultando na imagen que têm do recurso em questão e, por extensão, dos conservadores-restauradores.

Palabras-chave: Património cultural; Museu; Conservador-restaurador; Conservação preventiva; Difusão preventiva; Visitante.

\section{Communicate with visitors: a 'new' professional role for conservators-restorers}

Abstract: Heritage resources are more exposed than ever to visitors. For this reason, the transmission of cultural content is not enough: the present situation demands the inclusion of some messages able to foment a sustainable relation between the visitor and the resource. And this can best be achieved with the Preventive Difusion, a sensitizing strategy to inform and persuade visitors about the extreme fragility of the heritage resources.

In addition, this strategy is a magnificent tool at the service of the corporate identity of the heritage resources and of the conservators: usually, preventive conservation actions and policies are not clear to visitors. But if they are included into the visit discourse, the public receive an accurate vi- 
sion of the efforts done in that sense, and it allows them to have a clear idea of these efforts, leading to the image that they have about the resource in question and, by extension, of their conservators.

Keywords: Cultural heritage; Museum; Conservator-restorer; Preventive Conservation; Preventive Dissemination; Visitor.

Quien antes pensaba en "secreto", hoy tiene que pensar en "difusión"

Gaël de Guichen

\section{Introducción}

Si los visitantes son una variable esencial en la gestión de los museos y otros atractivos patrimoniales, está claro que habría que exprimir todas las posibilidades a nuestro alcance para que su interacción sea totalmente positiva.

La mejor forma de conseguir una relación plena, basada en el respeto y la comprensión, es involucrando al visitante. Nadie sentirá empatía por un museo o atractivo patrimonial si solo se le prohíben cosas y no se le explica nada.

En este cambio de paradigma de la relación museo/atractivo patrimonial-visitante, que debería progresar paulatina y progresivamente de la prohibición a la justificación y de la falta de información a la explicación puntual, los profesionales de la conservación-restauración ocupan un lugar de privilegio: la primera fila de una nueva gestión relacional.

¿Quién sino es el profesional que sabe el qué y el por qué de determinadas acciones que no es bueno que hagan los visitantes en el ecosistema museístico? ¿Quién sino es el profesional que sabe el qué y el por qué de determinadas realidades físicas y ambientales de ese mismo frágil ecosistema?

En este artículo se hablará exclusivamente de una estrategia de comunicación que pretende incidir en el momento en que interactúan visitantes y bienes culturales visitados. Merece la pena puntualizar ahora que, en paralelo, existen disciplinas como la Interpretación del patrimonio que tienen un objetivo similar (Morales 2008) o toda una corriente teórica con multitud de aplicaciones prácticas que tiene como objetivo la sensibilización sobre estos temas no solo de los visitantes, sino del conjunto de la sociedad (Ruiz de Lacanal 2012). Es decir, la educación del patrimonio basada en el conocimiento «para» el patrimonio (que se suma al conocimiento «sobre» y «a través» del patrimonio), que permite desarrollar actitudes y valores de conservación y respeto (Juanola, Calbó y Vallès 2005:27-28). Como también es necesario comentar que en estos aspectos de sensibilización sobre la fragilidad de los recursos patrimoniales y del nuevo rol de los visitantes en su conservación han sido pioneros los gestores de espacios naturales, los primeros en ser conscientes del papel fundamental de los visitantes en la protección del patrimonio natural, como acertadamente comenta Gaël de Guichen (Guillemard 2011:92).

Por ello, se comenzará por explicar qué es la Difusión preventiva, qué pretende y cuándo y cómo nace, para pasar a justificar la necesidad que los conservadores-restauradores la incluyan en su rutina laboral, acabando con algunas pistas para confeccionar píldoras de Difusión preventiva. 


\section{¿Qué es y qué pretende la Difusión preventiva? Definición y marco filosófico}

Antes de ofrecer una definición de Difusión preventiva, es obligado definir la disciplina en la que se circunscribe, la Conservación preventiva. Según G. de Guichen (1995: 5), la Conservación preventiva es:

"L'ensemble des actions destinées à assurer la sauvegarde (ou à augmenter l'espérance de vie) d'une collection ou d'un objet."

Concretando un poco más, según el ICOM-CC (International Council of Museums-Committee for Conservation, 2008) son:

"Todas aquellas medidas y acciones que tengan como objetivo evitar o minimizar futuros deterioros o pérdidas. Se realizan sobre el contexto o el área circundante al bien, o más frecuentemente un grupo de bienes, sin tener en cuenta su edad o condición. Estas medidas y acciones son indirectas - no interfieren con los materiales y las estructuras de los bienes. No modifican su apariencia."

Como una más entre las muchas y variadas medidas y acciones para conservar preventivamente un bien cultural tenemos la Difusión preventiva, la estrategia de sensibilización para informar y persuadir al público visitante de la extrema fragilidad de los recursos patrimoniales, con la intención de incidir en una actitud capaz de fomentar comportamientos respetuosos y colaborativos (Mateos, Marca y Attardi, 2011:8).

Una herramienta estratégica que, de forma independiente o integrada en el discurso general de mediación cultural (divulgativo o educativo), envía píldoras (léase mensajes) capaces de sensibilizar al público visitante de la fragilidad de los recursos patrimoniales, fomentando actitudes de respeto y comportamientos de buen uso (Nardi 1999; Guichen 2000; Ardemagni 2003; Ardemagni 2008). Las píldoras de Difusión preventiva vendrían a ser, ampliadas a todo tipo de públicos, lo que Nicole Gesché-Koning, presidenta del ICOM-CECA entre 1995-98 (International Council of MuseumsCommittee for Education and Cultural Action) Ilama los Cinq minutes pour l'éternité, aquellas pequeñas dosis sobre aspectos de conservación que los educadores de museos deberían integrar en sus actividades educativas destinadas a los más jóvenes (ICOM-CC 2007: 8).

Como una gran mayoría de visitantes de los bienes culturales activados como productos culturales no son conscientes de los muchos esfuerzos que se hacen para conservarlos y exponerlos de forma óptima (Gómez y Tapol 2009: 35), el objetivo esencial de la Difusión preventiva es conseguir su sensibilización, mediante recursos estrictamente comunicativos, sobre la fragilidad y los esfuerzos y dificultades para mantenerlos en buenas condiciones de conservación, potenciando así actitudes activas de respeto y protección. Como bien comenta Monica Ardemagni (Ardemagni 1997: 89), responsable hasta el 2004 de proyectos para la sensibilización del público del ICCROM (International Centre for the Study of the Preservation and Restoration of Cultural Property):

"Prevenir no quiere decir solamente tomar determinadas medidas para impedir el deterioro del bien, sino también informar al público que ese bien es frágil, que puede desaparecer para siempre y que necesita particular atención. La relación con el bien cultural se limita todavía al conocimiento de su existencia o de su importancia; hoy es necesario agregar otro elemento: la conciencia de su fragilidad." 
Un nuevo planteamiento que supone un cambio de paradigma sobre el papel y la responsabilidad de los visitantes. Utilizando el feliz juego de palabras ideado por el reputado especialista en conservación preventiva G. de Guichen (2000: 20), una nueva percepción que allá donde veía un visitante predador ahora verá un visitante protector:

"Pour beaucoup de professionnels du patrimoine, le public est source de revenu mais aussi ennemi du patrimoine: ils considèrent ce public comme un consommateur «prédateur». Pour assurer la survie du patrimoine culturel, cette mentalité doit absolument évoluer: de consommateur PRédaTEUR, le public doit devenir consommateur PRotecTEUR."

El propio G. de Guichen (1984: 233) ya avisaba de la pérdida que suponía desestimar la posible ayuda del visitante:

"Los profesionales de los museos se privan de una ayuda inestimable si no apelan a la colaboración que el público puede y debe brindar en la tarea de conservación del patrimonio. Deberían por el contrario utilizar todos los medios a su alcance para informar al público sobre la fragilidad de las colecciones. Y en esta tarea los educadores de los museos podrían cumplir una función de importancia capital."

Se considera a los visitantes como corderos que se han tenido que "calzar" una piel de lobo, obligados por unas activaciones patrimoniales donde en la mayoría de las situaciones no se les ha tenido en cuenta (Mateos 2006). Los visitantes no sólo pueden dejar de ser lobos depredadores, sino que pueden incluso ser auténticos protectores (Ardemagni 1997, 2003; Guichen 2000), obviamente si se piensa en ellos a la hora de planificar los productos patrimoniales, también desde el punto de vista comunicacional.

En definitiva, una estrategia comunicativa de probada solvencia para conseguir ese cambio de actitud y comportamiento, basada en dos verbos: justificar y explicar.

Justificar con determinadas recomendaciones el comportamiento que se les invita a tener durante la visita, colaborando en su uso correcto y respetuoso.

Explicar determinadas actuaciones o políticas de conservación preventiva que permiten visualizar de manera explícita el trabajo activo y continuado para conseguir su preservación.

Los productos patrimoniales, ya sean museos, centros expositivos, yacimientos arqueológicos o monumentos musealizados, verdaderos espacios de transmisión educativa y cultural, no deberían continuar utilizando exclusivamente medidas restrictivas para informar a sus usuarios de la actitud y comportamiento que se espera mantengan durante su visita. Las largas listas de prohibiciones pueden llegar a ser efectivas, pero sin lugar a dudas son nada o escasamente educativas. Cambiar el tono y explicar los motivos por los cuales se recomienda una determinada acción están más acordes con esos lugares de educación y cultura. Aunque bien es cierto que no se debe pecar de ingenuos como para creer que se deba abandonar totalmente la actual política de control y seguridad, cuando con bastante asiduidad nos asaltan y sobresaltan noticias de actos vandálicos perpetrados contra bienes culturales. Más bien lo que se propone es la utilización conjunta de ambas estrategias, la coercitiva y la educativa, planteando un futuro idílico (y quizá utópico) en que la primera ya no fuera necesaria.

Mensajes preventivos que ya se deberían enviar a los visitantes más jóvenes, uno de los públicos más importantes de los productos patrimoniales, integrándolos en las actividades didácticas que 
se les ofrecen. Y debería ser así por partida doble, ya que se les sensibiliza para el hoy y para el mañana: un niño o niña sensibilizados sobre estos temas será un adulto igualmente sensible.

Algo que ya se tiene muy en cuenta entre los profesionales de este área de la mediación cultural, como demuestra la organización inglesa Kids in Museums, que viene publicando desde 2003 un manifiesto donde se recogen una serie de recomendaciones para facilitar la visita de niños y familias a los museos (el eslogan del manifiesto es muy significativo: '20 ways to make a visit family friendly'), entre las que se recoge alguna en este sentido. Unas recomendaciones que, como se verá a continuación, han ido evolucionando en los diferentes manifiestos:

"Be interactive and hands on - where kids can touch objects, and learn to know what they are allowed to handle, and what they aren't (Kids in Museums 2007).

'Don't touch' is never enough. Say why. Use positive remarks like, 'Isn't that a great painting! Let's look at it together from further back.' Teach respect by explaining why some things shouldn't be touched. Direct to something nearby which can be (Kids in Museums 2010)."

En su última versión, de 2012, es interesante ver el comentario del que parte la décima recomendación 'It was really fun wearing the white gloves so we could pick things up':

"Say 'Please touch!' as often as you can. Everyone finds real objects awesome. Direct kids to things that can be handled. Teach respect by explaining why others can't" (Kids in Museums 2012).

\section{Apuntes sobre su corta historia}

El nacimiento y desarrollo de esta estrategia tiene nombre, apellido y fecha de natalicio: Gaël de Guichen durante los años ochenta del siglo XX.

Los planteamientos teóricos de G. de Guichen los asumiría como propios el ICCROM, que los incluiría en el articulado de sus estatutos a partir del año 1991. A partir de ese año la mencionada organización intergubernamental se compromete, mediante el punto «e» del primer artículo de sus estatutos, a: "encourager les initiatives tendant à créer une meilleure compréhension de la conservation et de la restauration des biens culturels" (ICCROM 2005).

Fruto de ese compromiso estatutario, el organismo internacional dará un verdadero impulso al tema, diseñando a partir de entonces iniciativas piloto como modelo a seguir para los gestores de los recursos patrimoniales de todo el mundo, además de difundir la temática entre estos profesionales mediante la celebración de conferencias y workshops y la publicación de manuales y libros. Una alianza en la que se trabaja desde hace ya algo más de veinte años, cuando se iniciaron proyectos dirigidos exclusivamente al público infantil, para ampliarse paulatinamente al público adulto y a determinados sectores profesionales que se pueden utilizar como canales para difundir masivamente el tema, como los medios de comunicación y los operadores turísticos (Blondé 2000; Pardo 2000; Grattan 2004; Ardemagni 2004; Ardemagni 2008).

Como muestra de los proyectos dirigidos a los jóvenes, resalta el primero de los impulsados por el ICCROM (en este caso junto al ICR, el Istituto Centrale del Restauro), Salvemos a Marco Aurelio, iniciado el año 1985 para sensibilizar a ese segmento de público del deterioro del patrimonio aprove- 
chando la restauración de la estatua ecuestre del emperador Marco Aurelio (Basile 1995; Blondé 2000: 117-119).

Para el público adulto, el ICCROM (también con la participación del ICR) seleccionó en 1997 como prueba piloto del proyecto Juntos velamos por nuestro patrimonio el sitio arqueológico de Ostia Antica en Roma, un laboratorio de pruebas pensado para sensibilizar a sus visitantes (Blondé 2000: 49-52).

Entre los preocupados por plantear la cuestión entre los profesionales de los medios de comunicación, se instauró el año 1991 el Media Save Art Award, pensado para premiar las mejores piezas periodísticas en prensa no especializada que tratasen los problemas de conservación del patrimonio cultural (Pardo, 2000; Ardemagni, 2007). Un premio al que se presentarían medios de comunicación españoles, consiguiendo que artículos como Bòsnia i Croàcia: l'herència de guerra (Jordi Zamora, El Temps, Valencia, 1997) y San Jerónimo de Buenavista: los expolios del monasterio sevillano más saqueado (Pablo Ferrand, $A B C$, Sevilla, 2001) recibiesen alguno de los premios (Ruiz de Lacanal 2002).

El último de los esfuerzos del organismo internacional se centró en conseguir la inclusión de mensajes explícitos sobre la fragilidad del patrimonio en las guías turísticas de gran tirada y difusión internacional, como las guías Lonely Planet, Michelin o Touring Club (Ardemagni 2004: 8). Para ello, se redactó el siguiente vademecum del turista responsable (ICCROM 2006):

\section{"Les directives du tourisme responsable}

Le patrimoine culturel est constitué de biens fragiles pouvant se détériorer à la fois lentement ou rapidement, en raison de phénomènes naturels et humains.

Même si cette détérioration ne peut pas être complètement arrêtée, votre comportement peut contribuer à en ralentir le processus.

\section{Contribuez à la préservation du patrimoine pour les générations à venir}

1. Acceptez les restrictions

Acceptez de bonne grâce certaines interdictions (ne pas toucher, ne pas photographier, ne pas courir) ou restrictions.

\section{Evitez de toucher}

Rappelez-vous que chaque contact, même le plus innocent effleurement, se transforme en menace lorsqu'il est répété par 1000, 10000, 100000 personnes.

\section{Portez des chaussures adaptées}

Pour éviter d'endommager les pierres ou mosaïques anciennes, portez des chaussures adaptées, et gardez talons hauts et semelles cloutées pour d'autres occasions.

\section{Les désastres du sac à dos}

Lorsque vous vous trouvez dans un espace clos et rempli de personnes, comme un tombeau ou une chapelle offrant des fresques, faites attention à votre sac à dos: il pourrait frotter contre les murs et détruire les fresques.

\section{Evitez l'escalade}

Les statues, les monuments et les murs anciens ont survécu à travers les siècles, et 
ils sont vieux et fragiles. Evitez de les escalader pour prendre des photos ou vous faire photographier.

\section{Stop aux graffitis}

Bien que le désir d'être immortel soit inhérent à la nature humaine, résistez à la tentation de gribouiller votre nom ou de dessiner sur les monuments et les bâtiments.

\section{Mosaïques manquantes}

Vous aimeriez remporter à la maison en guise de souvenir un morceau de la mosaïque que vous avez tant admirée? Combien de visiteurs ayant le même souhait faudra-t-il avant que la mosaïque disparaisse pour toujours?

\section{Achetez responsable}

Les personnes dérobant les biens culturels de touts types ouvrent la voie au pillage systématique et au trafic illicite d'œuvres d'art. Faites attention si vous achetez des objets dont la provenance est inconnue.

\section{Ne salissez pas}

Les sites historiques et archéologiques ne sont pas des poubelles. Emportez vos détritus avec vous!

\section{Respectez le silence}

Soyez respectueux de l'atmosphère de certains lieux qui invitent à la méditation et au silence, et évitez la pollution sonore (cris, klaxons, radios, téléphones mobiles etc.). tous."

La protection de notre patrimoine culturel dépend de chacun de nous, et nous concerne à

Prácticamente de forma paralela al ICCROM, el CCA (Centro di Conservazione Archeologica di Roma) inició una línea de trabajo propia que incluía esa relación comunicativa con el público, basada en la apertura de las obras de restauración del Arco de Septimio Severo en el Foro Romano (el año 1985) o del Atrio del Museo Capitolino (entre 1990 y 1993), entre otros muchos (Nardi, 1995: 10). Quizá uno de sus proyectos de sensibilización más interesantes fue Coliseo Mío, llevado a cabo en el Coliseo romano a finales de los años 90, que contaba con seis programas de sensibilización pensados para seis tipos diferentes de público: Coliseo mío, iqué bello eras!, Coliseo mío, iqué bajo has caído!, Coliseo mío, iqué te están haciendo!, Coliseo mío, quiero conocerte, Coliseo mío, te queremos mucho y Coliseo mío, eres mío (Nardi 1999: 45-50).

Esas experiencias lideradas por el ICCROM llegarían años más tarde a España. En nuestro país, fue pionero el Museo Arqueológico de Granada con el proyecto que se desarrolló a partir del llamado Guerrero de Baza. Un proyecto dirigido al público infantil inspirado en el del Marco Aurelio desarroIlado años antes por el ICCROM y el ICR (San Martín et al. 2001).

\section{¿Por qué los conservadores-restauradores tienen que apostar por la Difusión preventiva?}

El objetivo preventivo de la Difusión preventiva ya ha quedado claro, por lo que se comentará a continuación otro de sus objetivos: el de notoriedad e imagen de los profesionales que tienen la responsabilidad de velar por su óptima conservación, entre los que por su relevancia hay que mencionar a los conservadores-restauradores. 
Como esas pistas ocultas que nos regala el mundo de la música, la inclusión de mensajes que explican determinadas actuaciones o políticas de conservación preventiva, que permiten visualizar de manera explícita el trabajo activo y continuado para conseguir su preservación, es una magnífica herramienta al servicio de la imagen pública del equipamiento y de sus gestores.

Para incidir en la opinión pública de los visitantes es obligado establecer una comunicación con ellos, modulando estratégicamente lo que se les quiere contar y cómo se les quiere contar. Lo que no se conoce no se valora. Y está claro que las instituciones patrimoniales y sus gestores hacen un intenso trabajo para asegurar su pervivencia que bien merece del reconocimiento público.

Integrar en el relato que se traslada a los visitantes alguna de esas cuestiones del día a día que normalmente no se explicitan, permite tangibilizar claramente los esfuerzos en ese sentido, lo que favorece la construcción de la imagen mental que tendrán del equipamiento en cuestión y, por extensión, de sus gestores y de sus conservadores-restauradores. Necesariamente mucho más positiva que en la situación anterior en la que ese tipo de información se eludía.

Una forma contundente de hacerlo es abandonar los talleres de restauración y realizar el proceso de restauración de una pieza en las mismas salas de exposición o trasladar allí directamente el taller (Figura 1).

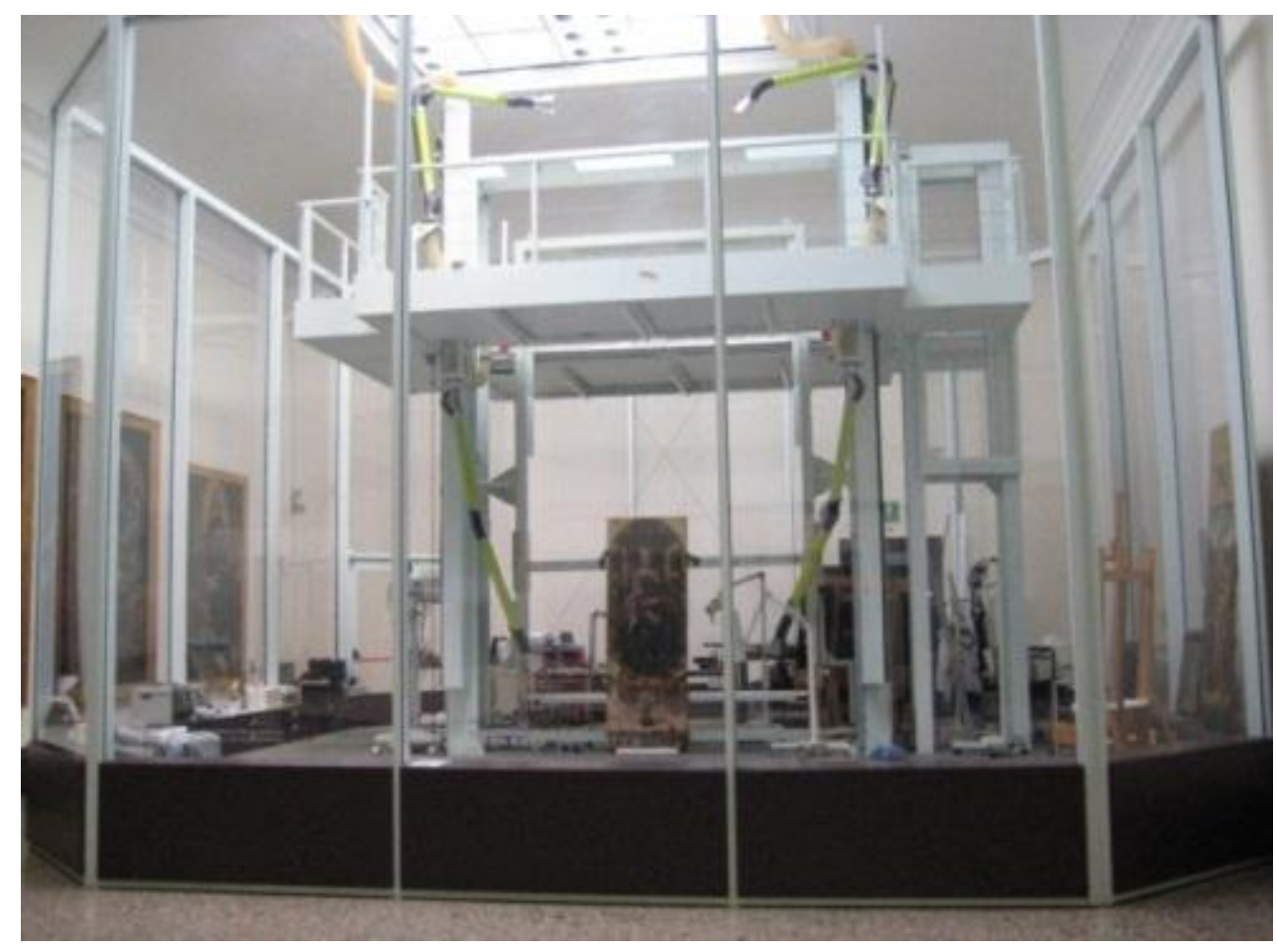

Figura 1. Una forma rotunda para que el visitante conozca el trabajo de los conservadores-restauradores es trasladar el taller a las salas, como en este caso de la Pinacoteca di Brera de Milán (fuente: Santos M. Mateos).

Otra forma, más sutil, es informar a los visitantes de determinadas medidas o acciones de conservación preventiva. Por ejemplo, en una exposición, explicar que la intensidad lumínica es menor en la zona donde se muestran tejidos u obras sobre papel para asegurar así la correcta conservación de los frágiles objetos realizados en esos materiales. Algo que no solo sirve para que sean conscien- 
tes del cuidado que se presta a las piezas, sino que además corrige la percepción negativa que algunos visitantes puedan tener al ver unos objetos con una iluminación tan escasa.

\section{Algunas pistas para producir píldoras}

Sirvan como ejemplo de lo que se habla los mensajes que se encontraban los visitantes de la Tate Britain de Londres antes de acceder a la exposición temporal Rude Britannia: British Comic Art (9 de junio-5 de septiembre del 2010), o los del Museo de las Escuelas Menores de la Universidad de Salamanca antes de acceder a la sala que conserva el llamado "cielo de Salamanca", la pintura mural que pintó en la segunda mitad del siglo XV el pintor Fernando Gallego para ilustrar la antigua bóveda de la biblioteca del Estudio universitario salmantino. Dos casos que ilustran otras tantas formas de explicar lo mismo pero utilizando para ello diferentes fórmulas comunicativas.

En el cartel que los visitantes se encontraban al iniciar la visita a la exposición de la pinacoteca londinense, se les explicaba puntualmente esta cuestión (Figura 2). Y se hacía mediante dos mensajes: un breve texto 'The light levels are kept at a low level throughout this exhibition, for the protection of Works of art' (Los niveles de luz se mantienen en un nivel bajo en esta exposición, para la protección de las obras de arte) y un pictograma con la silueta de una bombilla que incluía el texto 'Low light levels' (Bajos niveles de luz).

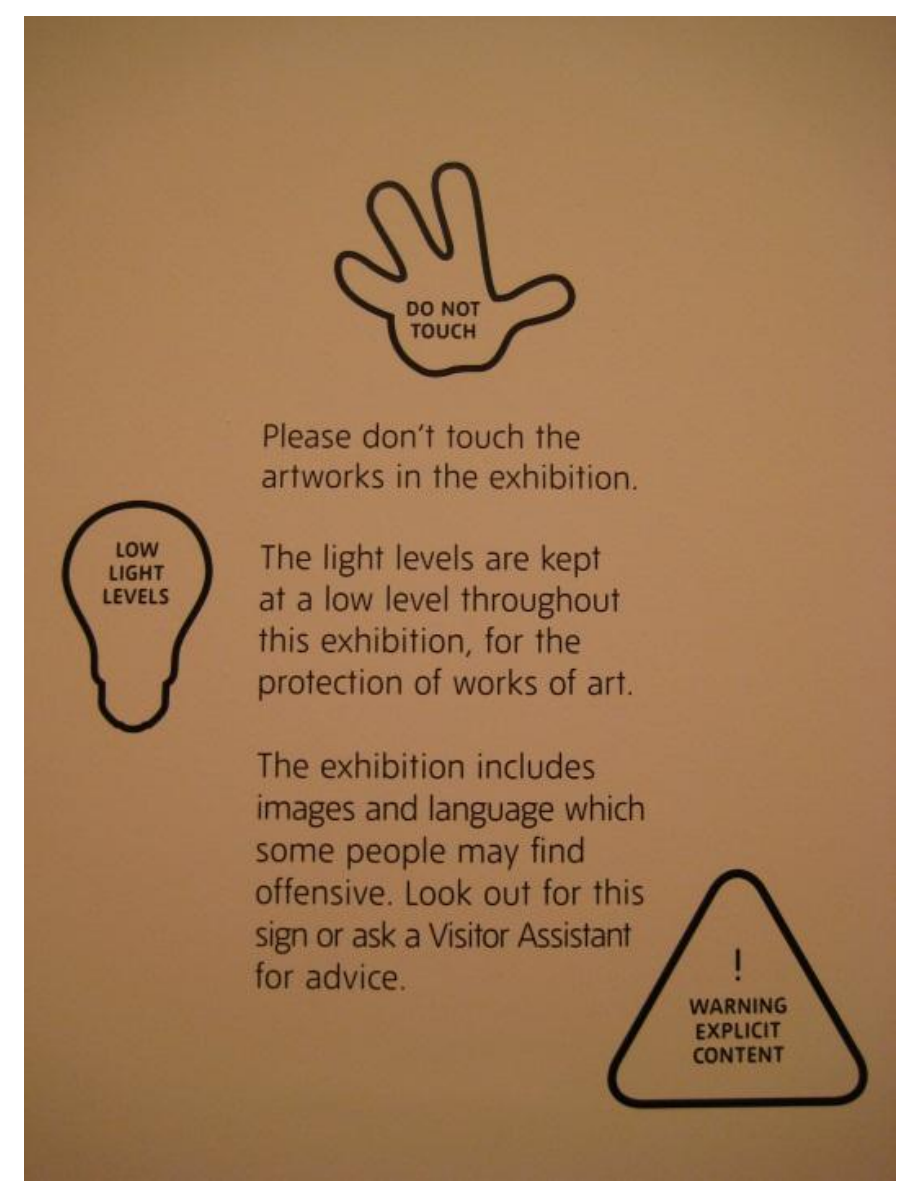

Figura 2. El cartel con los mensajes que informaban puntualmente a los visitantes de las condiciones lumínicas especiales de la exposición (fuente: Santos M. Mateos). 
En el caso salmantino (Figura 3) se va un poco más allá, al construirse un mensaje que explica lo mismo pero utilizando otros recursos para conseguirlo. El texto, trilingüe, dice lo siguiente: 'Esta visita precisa un mínimo de cinco minutos de su tiempo para acomodarse al ajuste luminoso del espacio. Es una medida de conservación preventiva que garantiza la estabilidad de la pintura titulada "el cielo de salamanca" (1473) que es obra del pintor Fernando Gallego (Salamanca 1440 ¿? 1507)'.

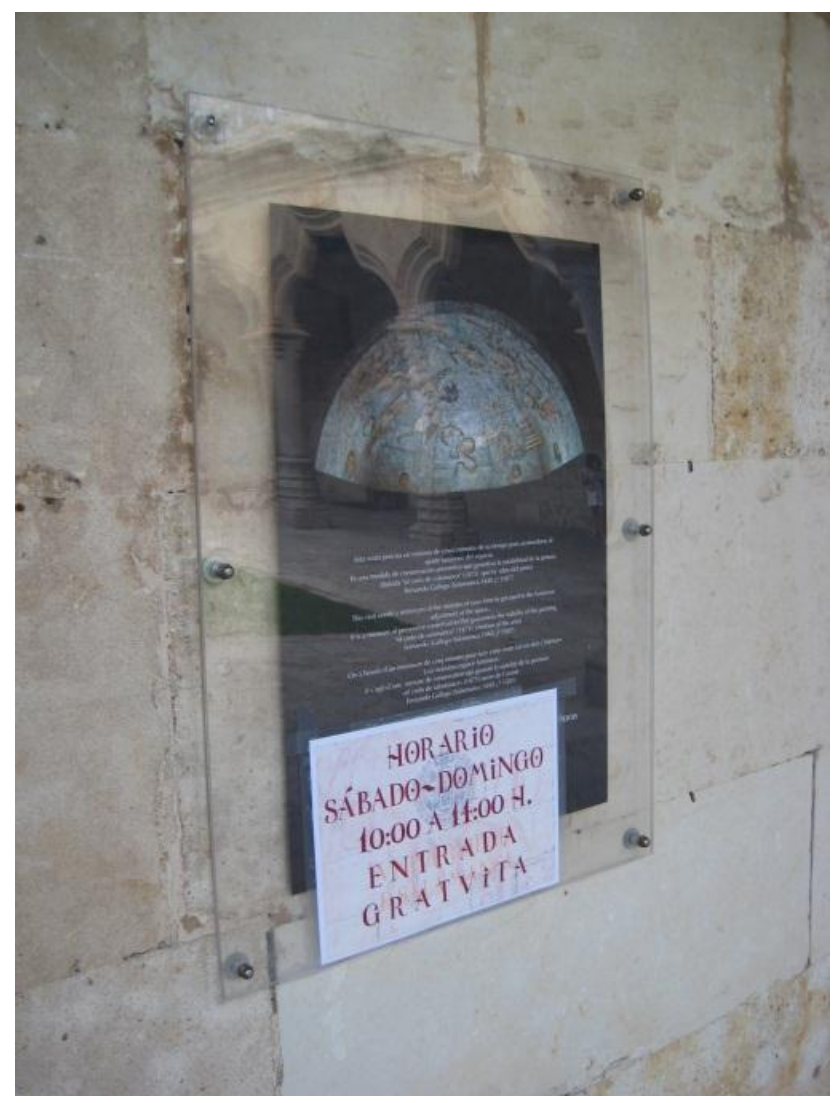

Figura 3. El cartel que avisa a los visitantes del tiempo que tardará su vista en adaptarse a las condiciones lumínicas del espacio en el que se expone "el cielo de Salamanca" (fuente: Santos M. Mateos).

Contrariamente a los dos casos descritos, cuando no se comunican este tipo de detalles (desgraciadamente algo más usual de lo que sería deseable), se está perdiendo una oportunidad de oro para explicarle al visitante el trabajo que se hace en un museo tras las bambalinas, dejando que además pueda llegar a pensar cosas negativas sobre algo que es totalmente necesario y positivo. ¿O es que alguien piensa que pasar de una zona iluminada con intensidad a otra en penumbra no es percibido negativamente por el visitante que no conoce las razones técnicas para que así sea?

Además de incluir este tipo de información que explica determinadas decisiones que atañen a la correcta conservación de los objetos y espacios patrimoniales, no hay que olvidar que el concepto de conservación preventiva también puede aplicarse al propio visitante. En este sentido, se trataría de todas aquellas medidas y acciones que tengan como objetivo evitar o minimizar futuros accidentes, asegurando una visita segura y placentera. Si como defendemos, tan importante es el patrimonio cultural como los visitantes que lo disfrutan, también habría que tenerlo en cuenta. 
Por ejemplo, es muy buena idea avisar a los visitantes de la Torre de Londres de las precauciones a tomar ante los míticos cuervos que viven en ella (Figura 4), lo que seguro que ha evitado, evita y evitará más de un susto o una desgracia, a la par que demuestra a los visitantes que se tiene en cuenta su confort durante su estancia en las instalaciones. Es un caso especialmente interesante, ya que permite ilustrar las dos formas de dosificación de las píldoras de Difusión preventiva: en formato monodosis -léase de forma independiente- (Figura 4) o en dosis integradas en el relato divulgativo o educativo que le descubre al visitante los secretos del producto patrimonial, en este caso la Torre de Londres (Figura 5).

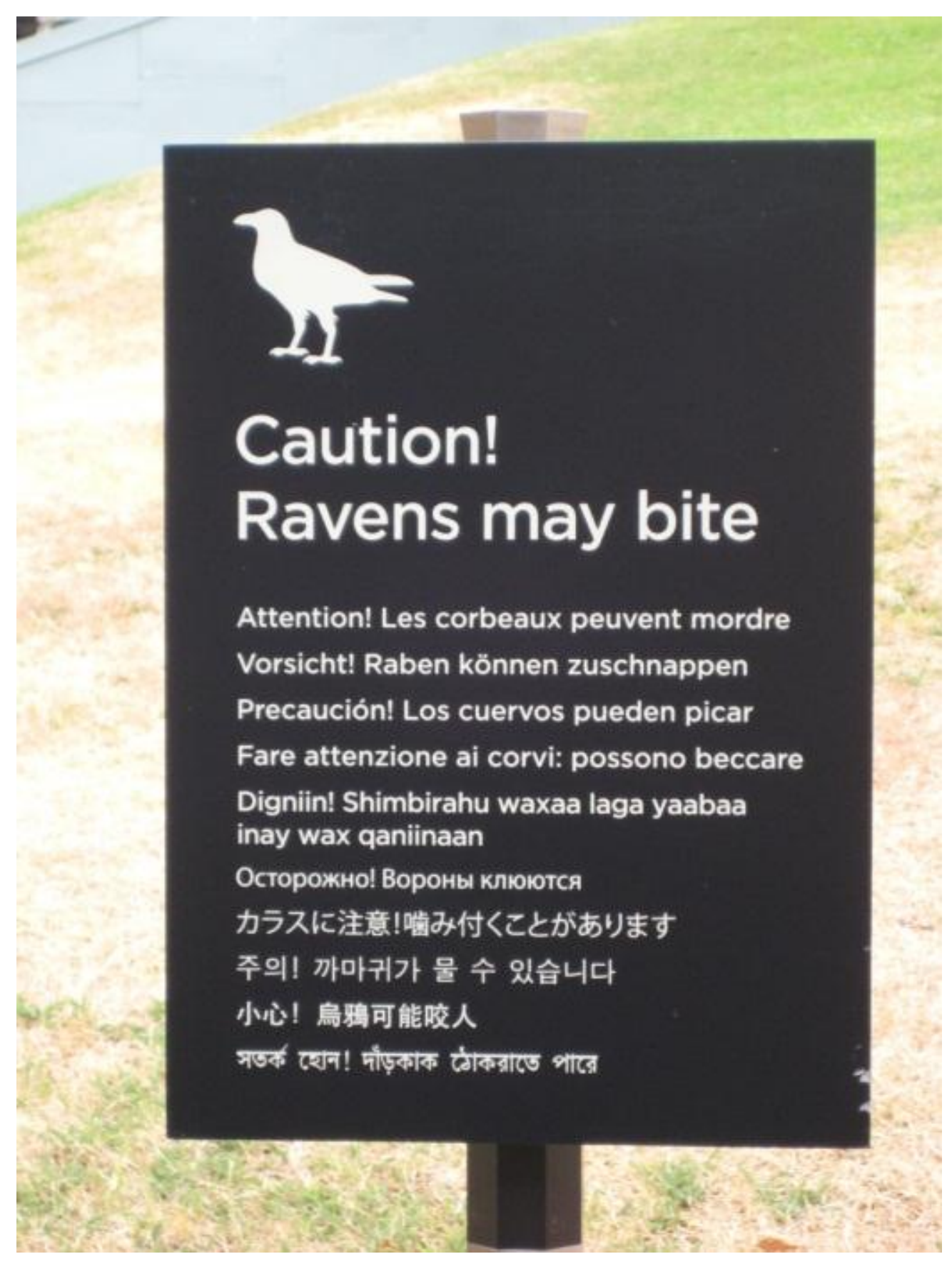

Figura 4. En varios idiomas, el cartel avisa a los visitantes de la Torre de Londres del peligro de los aparentemente inofensivos cuervos (fuente: Santos M. Mateos). 


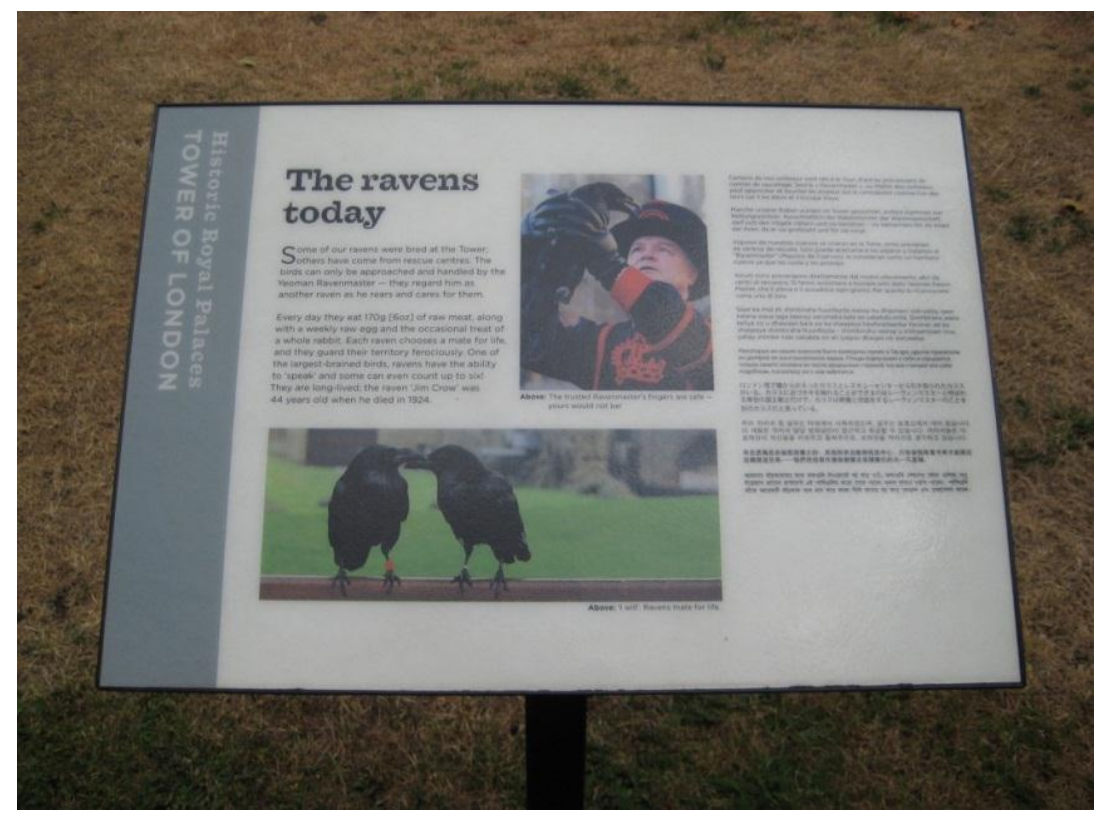

Figura 5. En uno de los carteles divulgativos que explican la leyenda de los cuervos, se aprovecha para alertar que sólo el Yeoman Ravenmaster puede acercarse a ellos (fuente: Santos M. Mateos).

\section{Conclusión}

Por todo lo comentado, está claro que los conservadores-restauradores deberían incluir esa 'nueva' expertise comunicativa entre sus muchos y variados conocimientos y habilidades profesionales. No se habla de un nuevo experto y de una nueva especialidad, se habla de incorporar la cuestión comunicativa entre las competencias de los profesionales de la conservación y la restauración.

Para conseguirlo, no estaría mal comenzar por incorporarla decididamente en la formación específica de los futuros profesionales, en nuestro país el Grado en Conservación y Restauración de Bienes Culturales. Algo que se contempla de manera ambigua en el Real Decreto 635/2010, de 14 de mayo que regula el contenido básico de esta enseñanza superior (BOE 2010). No tiene cabida en ninguna de las asignaturas del programa docente (ni entre aquellas que conforman el núcleo de formación básica ni entre las de especialización), aunque curiosamente sí se tiene en cuenta entre las competencias (transversales y generales) que debe poseer el alumnado al finalizar los estudios, concretamente:

\section{Competencia transversal:}

Contribuir con su actividad profesional a la sensibilización social de la importancia del patrimonio cultural, su incidencia en los diferentes ámbitos y su capacidad de generar valores significativos.

\section{Competencia general:}

Tener capacidad para obtener, presentar y difundir información sobre los bienes culturales y la metodología de los procesos de conservación-restauración." 
Si los responsables de la gestión de museos y atractivos patrimoniales quieren conseguir proyectar esa imagen positiva en la mente de los visitantes, la implicación de los conservadoresrestauradores debe ser clara y directa. De forma que, en los procesos de difusión que se establecen con los visitantes de la institución, su papel pase del mayoritariamente de reparto que tienen en la actualidad al de protagonistas principales.

Aquello que no se comunica no se conoce y, por tanto, no se valora. Y para ser visibles, para pasar de un papel a otro, los conservadores-restauradores tendrían que exigir su participación en la construcción del relato que se traslada a los visitantes, cerciorándose así de la inclusión de esas pequeñas píldoras de Difusión preventiva que explican su trabajo. Parafraseando a N. Gesché-Koning, asegurando sus cinco minutos para la eternidad.

Por tanto, la labor de un conservador-restaurador no debería limitarse a la tradicional vigilancia de los recursos patrimoniales bajo su responsabilidad, pues tendría que extenderse más allá, llegando a incidir directamente, mediante la comunicación, en los usuarios de esos bienes culturales. Seguramente eso ayudará a que se valore socialmente y con justicia su papel capital en la gestión de nuestro patrimonio cultural.

\section{Bibliografía}

ARDEMAGNI, M. (1997). "La conservación preventiva y el gran público". En Actas del Coloquio Internacional sobre la conservación preventiva de bienes culturales, Hidalgo, J. M. (coord.). Vigo: Excma. Diputación provincial de Pontevedra, 89-104.

ARDEMAGNI, M. (2003). "¿Público predador o público protector? Cómo involucrar al público en la conservación del patrimonio", Mus-A. Revista de las instituciones del patrimonio histórico de Andalucía, 2: 99-103.

ARDEMAGNI, M. (2004). “iAtención visitante!", ICCROM Boletín, 30: 8.

ARDEMAGNI, M. (2007). "Patrimonio y público. La labor de sensibilización a través de los medios de comunicación". En Patrimonio cultural y medios de comunicación, DD. AA. Sevilla: Consejería de Cultura, Junta de Andalucía, 71-79.

ARDEMAGNI, M. (2008). "El público y la conservación del patrimonio". En La comunicación global del patrimonio cultural, Mateos Rusillo S. M. (coord.). Gijón: Trea, 111-129.

BASILE, G. (1995). "Les enfants peuvent aussi contribuer à la sauvegarde des œuvres d'art", Cahiers d'étudeStudy Series, París: ICOM-International Committee for Conservation, 1:11-12.

http://archives.icom.museum/study_series_pdf/1_ICOM-CC.pdf. [Consulta: 27/04/2012].

BLONDÉ, A. (ed.) (2000). Jeunes et sauvegarde du patrimoine. Cahier de sensibilisation des jeunes à la fragilité et à la conservation du patrimoine /Youth and the Safeguard of Heritage. Approaches to raising youth awareness of the fragility and the conservation of cultural heritage. Roma: ICCROM.

BOE (2010). Real Decreto 635/2010, de 14 de mayo, 137: 48548-48564.

GÓMEZ, M. , TAPOL, B. de (2009). "Medio siglo de Conservación Preventiva. Entrevista a Gaël de Guichen", Geconservación, 0: 35-44.

GRATTAN, N. (2004). ICCROM \& Public Advocacy. Roma: ICCROM. http://www.iccrom.org. [consulta: $16 / 03 / 2012]$.

GUICHEN, G. de (1984). “Enseñar a conservar el patrimonio”, Museum, 144 (xxxVI, 4): 232-233. 
GUICHEN, G. de (1995). "La conservation préventive: un changement profond de mentalité", Cahiers d'étudeStudy Series, París: ICOM-International Committee for Conservation, 1:4-6.

GUICHEN, G. de (2000). "Introduction. Notre patrimoine est fragile: protégeons-le!". En Jeunes et sauvegarde du patrimoine. Cahier de sensibilisation des jeunes à la fragilité et à la conservation du patrimoine/Youth and the Safeguard of Heritage. Approaches to raising youth awareness of the fragility and the conservation of cultural heritage, Blondé, A. (ed.). Roma: ICCROM, 13-23.

GUILLEMARD, D. (2011). “Conservation préventive, le témoignage d'un pionnier: entretien avec Gaël de Guichen", Technè, 34: 87-94.

ICCROM (2005). Statuts de I'ICCROM.

http://www.iccrom.org/fra/00about_fr/00_01govern_fr/statutes_fr.shtml. [consulta: 15/04/2012].

ICCROM (2006). Les directives du tourisme responsable.

http://www.iccrom.org/fra/05advocacy_fr/05_01raising_fr/01raisingtour_fr.shtml. [consulta: 15/04/2012].

ICCROM (2008). Terminology to characterize the conservation of tangible cultural heritage. http://www.icomcc.org/242/about-icom-cc/what-is-conservation/. [consulta: 15-06-2012].

ICOM-CC (2007). "Rapport de la séance de l'ICOM-CC à la Conférence générale de l'ICOM à Vienne", Bulletin, 26: 07-09.

JUANOLA, R.; CALBÓ, M.; VALLÈS, J. (2005). Educació del patrimoni: visions interdisciplinàries. Arts, cultures, ambient. Girona: Institut del Patrimoni Cultural de la Universitat de Girona.

KIDS IN MUSEUMS (2007). The Manifesto. http://www.kidsinmuseums.org.uk/wpcontent/uploads/2007/08/kids-in-museums-manifesto.pdf. [consulta: 16/04/2012].

KIDS IN MUSEUMS (2010). Kids in Museums Manifesto 2010. http://www.keepandshare.com/doc/2332449/kimmanifesto-a4-low-res-pdf-october-28-2010-10-36-am-854k?da=y. [consulta: 16/04/2012].

KIDS IN MUSEUMS (2012). Kids in Museums Manifesto 2012. http://www.kidsinmuseums.org.uk/manifesto/. [consulta: 16/04/2012].

MATEOS, S. M. (2006). "Corderos con piel de lobo: estrategias comunicativas para desenmascarar a los usuarios del patrimonio cultural". En Congreso Internacional sobre Gestión Turística de Patrimonio Cultural. Marchena (Sevilla): texto inédito.

MATEOS, S. M.; MARCA, G.; ATTARDI, O. (2011). "Sensibilizando al visitante: la Difusión preventiva", Miradas desde la copa. e-Revista de Comunicación y Patrimonio cultural, 3: 5-16. http://www.comunicacionpatrimonio.net/e-revista/. [consulta: 12/12/2011].

MORALES MIRANDA, J. (2008). "El sentido y metodología de la interpretación del patrimonio". En La comunicación global del patrimonio cultural, Mateos Rusillo, S. M. (coord.). Gijón: Trea, 53-77.

NARDI, R. (1995). "Open-heart Restoration: Raising the Awareness of the Public", Cahiers d'étude-Study Series, París: ICOM-International Committee for Conservation, 1: 09-11.

http://archives.icom.museum/study_series_pdf/1_ICOM-CC.pdf. [consulta: 27/04/2012].

NARDI, R. (1999). "Implicar al público: un nuevo enfoque de la educación para la conservación", Museum International, 201:44-50. 
PARDO, G. (ed.) (2000). Presse et sauvegarde du patrimoine. Recueil d'articles de presse abordant le thème de la fragilité du patrimoine et de sa conservation / The Press and the Safeguard of Heritage. Collection of press articles on the subject of the fragility of cultural heritage and its conservation. Roma: ICCROM.

RUIZ DE LACANAL, M. D. (2002). "Dos artículos referidos a España entre los premiados en el Premio Media Save Art", Revista de Restauración y Rehabilitación. Revista Internacional del Patrimonio Histórico, 62: 13.

RUIZ DE LACANAL, M. D. (2012). "La educación para la conservación del patrimonio cultural. Una educación para todos los públicos". En Actas del IV Congreso Internacional de Educación Artística y Visual. Arte, educación y cultura. Aportaciones desde la periferia. Jaén: Red Sociedad, Arte y Gestión cultural.

SAN MARTíN, C. et al. (2001). "Sensibilizar para conservar: una experiencia con público infantil en un museo arqueológico", PH. Boletín del Instituto Andaluz del Patrimonio Histórico, 34: 138-145.

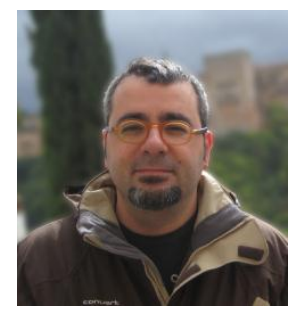

Santos M. Mateos Rusillo

santos.mateos@uvic.cat

Historiador del arte, es profesor titular de la Universidad de Vic (Barcelona).

Desde el 2009 es editor del portal web Miradas desde la copa. Portal de Comunicación y Patrimonio Cultural: www.comunicacionpatrimonio.net.

Artículo recibido el 03/05/2012

Artículo aceptado el 13/07/2012 